\title{
CASE REPORT OF A PATIENT WITH RARE P.ARG136CYS (APOE2*) MUTATION IN APOLIPOPROTEIN E GENE
}

\author{
${ }^{1}$ Jana Lisyova, ${ }^{1}$ Jan Chandoga, ${ }^{2}$ Stanislav Oravec, ${ }^{3}$ Dana Kantarska and ${ }^{1}$ Daniel Böhmer \\ ${ }^{1}$ Institute of Medical Biology, Genetics and Clinical Genetics, \\ Faculty of Medicine, Comenius University and University Hospital, Bratislava, Slovakia \\ ${ }^{2}$ Department of Internal Medicine, Faculty of Medicine, \\ Comenius University and University Hospital, Bratislava, Slovakia \\ ${ }^{3}$ Department of Medical Genetics, F.D. Roosevelt University Hospital, Banska Bystrica, Slovakia
}

Received 2013-11-09; Revised 2013-11-14; Accepted 2014-01-09

\begin{abstract}
Apolipoprotein E (apoE) is a polymorphic glycoprotein associated with plasma lipoproteins and plays an essential role in the metabolism and clearance of plasma lipids. ApoE can represent the major genetic risk factor in development of premature atherosclerosis, coronary heart disease and late-onset familial and sporadic Alzheimer's Disease (AD). We present a case report of a 51-year old male with suspected AD. After the routine biochemical tests, we performed a Lipoprint System analysis for evaluation of lipoprotein changes and subsequently the molecular-genetic analysis (PCR-RFLP, sequence analysis) of APOE gene. Moderate changes were seen in plasma lipid parameters-an increase of triacylglycerols and a drop of HighDensity Lipoproteins (HDL). Lipoprint System assay revealed a shift of lipoproteins to less dense fractions mainly to Very-Low-Density Lipoproteins (VLDL). The presence of a rare APOE mutation p.Arg136Cys has been detected in patient with severe short-term memory problems and symptoms of dementia. Our case report may open a discussion on possible linking of this rare genotype to neurological pathology and justifying the impact of apoE genotype on lipoprotein metabolism and AD.
\end{abstract}

Keywords: Apolipoprotein E Isoforms, Lipoprint System, PCR-RFLP, Sequence Analysis, p.Arg136Cys

\section{INTRODUCTION}

The metabolism of plasma lipoprotein particles is influenced and regulated by different types of apolipoproteins that are involved in the transport and redistribution of lipids among cells and tissues (Irshad and Dubey, 2005).

Apolipoprotein E (apoE) is a plasma 34 kilodaltons (kDa) glycoprotein composed of 299 amino acids (Elmadbouh et al., 2013) and plays an important role in the metabolism and clearance of plasma lipids (Elliott et al., 2010). ApoE has a critical role in the metabolism of chylomicrons and very-low-density lipoprotein (VLDL) remnants of human plasma. ApoE affects the binding of these lipoproteins to Low-Density
Lipoprotein (LDL) receptors and to receptors specific for chylomicron remnants, too. Three most common apoE isoforms occur and they are distinguished by a single amino acid substitution (Cys/Arg) at position 112 and 158. As a consequence, three apoE alleles signed $\varepsilon 2$ (cysteine in both positions), $\varepsilon 3$ (cysteine in position 112 and arginin in position 158), $\varepsilon 4$ (arginin in both positions) are present in humans and generate six possible genotypes (Cedazo-Minguez, 2007; Ward et al., 2009). These apoE isoforms differ in physiological function in respect to binding affinity to LDL receptors. Positively charged amino acid residue (arginine) at position 158 is responsible for normal apolipoprotein-receptor interaction in apoE3 and apoE4 isoforms while apoE2 isoform containing

Corresponding Author: Jana Lisyova, Institute of Medical Biology, Genetics and Clinical Genetics, Faculty of Medicine, Comenius University and University Hospital, Bratislava, Slovakia 
cysteine is less effective in binding (Zhong and Weisgraber, 2009).

A frequency and occurence of particular isoforms and genotypes varies between different populations and mainly between ethnics (Burman et al., 2009). More than thirty less frequent APOE gene variants have been described worldwide (Drenos and Kirkwood, 2010). Apos3 allele is the most frequent in Caucasian population $(60-80 \%)$.

Polymorphisms and rare mutations of APOE gene influence functions of apoE protein and can result in hyperlipidemia (hypertriglyceridemia, hyperlipoproteinemia type III), atherosclerosis and Alzheimer's Disease (AD). Carriers of different genotypes and thus of different apoE isoforms show distinct incidence of coronary artery disease (CAD), premature atherosclerosis and stroke (Drenos and Kirkwood, 2010). ApoE4 isoform represents the major genetic risk factor in development of these patologies as soon in late-onset familial and sporadic $\mathrm{AD}$ in Caucasians (Coon et al., 2007; Sando et al., 2008; Crean et al., 2011). Contrary, apoE2 isoform is responsible for lower risk of $\mathrm{AD}$ development. In general, carriers of $\varepsilon 4$ allele have higher total cholesterol levels while individuals carrying the $\varepsilon 2$ allele have lower cholesterol than those carrying the most frequent $\varepsilon 3 \varepsilon 3$ genotype (Hubacek et al., 2010).

APOE gene was mapped to chromosome 19 (Bertram et al., 2010), consists of 4 exons and is mainly exprimed in the liver, kidneys, lungs, spleen, skin, brain and various cells such as macrophages (Gafencu et al., 2007).

\section{MATERIALS AND METHODS}

Lipid parameters were determined in the patient's plasma by routine biochemical methods. For acquisition of detail lipoprotein pattern, the Lipoprint System (Quantimetrix Corporation) was used. It is an accurate, inexpensive and easy-to-use high resolution diagnostic test for plasma lipoproteins which are not routinely tested by other methods and it is the first and only Food and Drug Administration (FDA)-cleared methodology for the identification of LDL subfractions. This test provides results concerning lipoprotein classes and their subfractions: VLDL, three subfractions of Intermediate Density Lipoproteins (IDL), seven subfractions of LDL and fraction of High-Density Lipoproteins (HDL) (Fig. 3 and 4). The most important feature is that lipoprint allows to distinguish and to quantify the atherogenic
(VLDL, IDL1-2, LDL3-7) and antiatherogenic (IDL3, LDL1-2, HDL) lipoproteins. This method is based on linear polyacrylamide gel electrophoresis (Fig. 1). The separation of various lipoprotein fractions is achieved by sieving effect of the gel matrix (Hoefner et al., 2001) and is comparable with the ultracentrifugation as a reference method for lipoprotein separation (Sawle et al., 2002; Ping et al., 2005).

For the analysis, $25 \mu \mathrm{L}$ of fasting serum or plasma was used (refrigerated for up to 7 days). The sample was loaded into a gel tube, $200 \mu \mathrm{L}$ of loading gel was added and mixed. After $30 \mathrm{~min}$ of polymerization of loading gel, the electrophoresis at $3 \mathrm{~mA} / \mathrm{gel}$ tube for 1 hour was performed. The gel tubes were scanned and the analysis was made by LipoWare analysis software that automatically identified and quantified the relevant lipoprotein bands.

To qualify the hereditary determinants of patient's lipoprotein phenotype, we revealed apoE isoform by molecular-genetic analysis. A genomic DNA was isolated from uncoagulated whole blood by NucleoSpin Blood QuickPure columns (Macherey-Nagel). ApoE genotype was identified by Polymerase Chain ReactionRestriction Fragment Length Polymorphism (PCRRFLP) analysis using HhaI restriction endonuclease (Gregorio et al., 2013). PCR reaction was performed in $25 \mu \mathrm{L}$ reaction volume containing $10 \times$ Thermo Start PCR Buffer (Thermo Scientific), $200 \mu \mathrm{mol} \mathrm{L} \mathrm{L}^{-1}$ deoxyribonucleotides (dNTP) each (Fermentas), 25 mmol $\mathrm{L}^{-1} \quad \mathrm{MgCl}_{2} \quad$ (Thermo Scientific), $10 \%$ Dimethylsulfoxide (DMSO) (Finnzymes), $0.3 \mu \mathrm{mol} \mathrm{L}^{-1}$ forward and reverse primer each (ApoE-1 and ApoE-2, respectively) designed by our laboratory (Merck) and $0.625 \mathrm{U}$ of Thermo Start PCR Polymerase (Thermo Scientific). The reaction conditions consist of the following steps: $95^{\circ} \mathrm{C}$ for $15 \mathrm{~min}, 38$ cycles of $94^{\circ} \mathrm{C}$ for $30 \mathrm{sec}, 69^{\circ} \mathrm{C}$ for $20 \mathrm{~seconds}, 72^{\circ} \mathrm{C}$ for $1 \mathrm{~min}$ followed by a $10 \mathrm{~min}$ extension at $72^{\circ} \mathrm{C}$ in Mastercycler ep gradient $\mathrm{S}$ (Eppendorf).

After amplification, 227 bp PCR fragment was digested by HhaI (Fermentas) for $1 \mathrm{~h}$ at $37^{\circ} \mathrm{C}$. This restriction endonuclease recognizes double-stranded DNA in GCGC palindromic sequences and gives the typical restriction band pattern for all genotypes (Fig. 2). The restriction fragments were then visualized on Spreadex EL 300 Mini gel (Elchrom Scientific).

The exact nucleotide position was confirmed by direct sequencing on automated genetic analyser ABI PRISM 310 (Applied Biosystems) using GeneScan-500 TAMRA Size Standard (Applied Biosystems). 
Jana Lisyova et al. / OnLine Journal of Biological Sciences 14 (1): 36-43, 2014

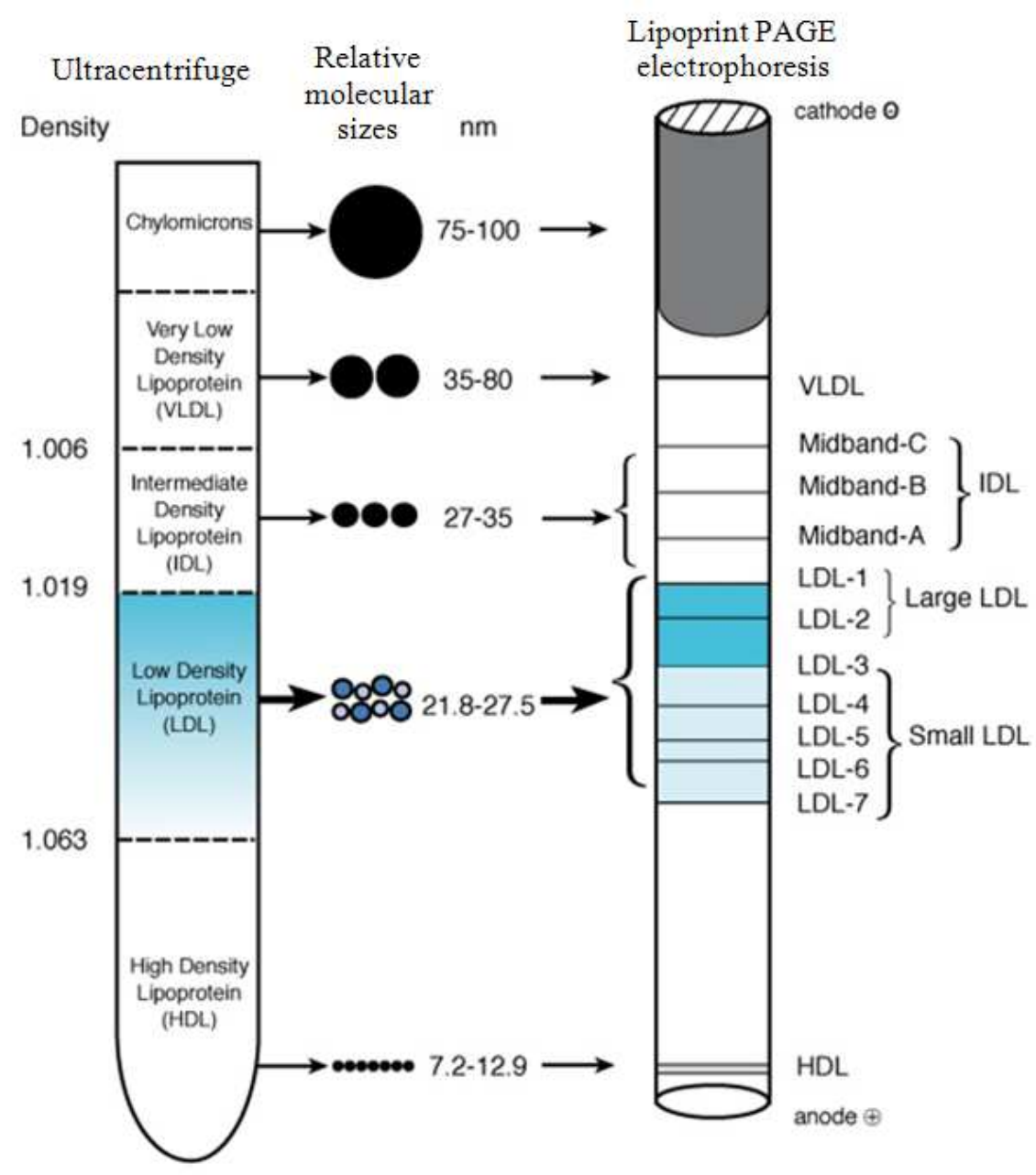

Fig. 1. Comparison of results of lipoprotein fractions acquired by Lipoprint System and continuous gradient ultracentrifugation (http://www.eurobio-mcv.fr/images/Image/File/Lipoprint/Ultracentri et Lipoprint.jpg)

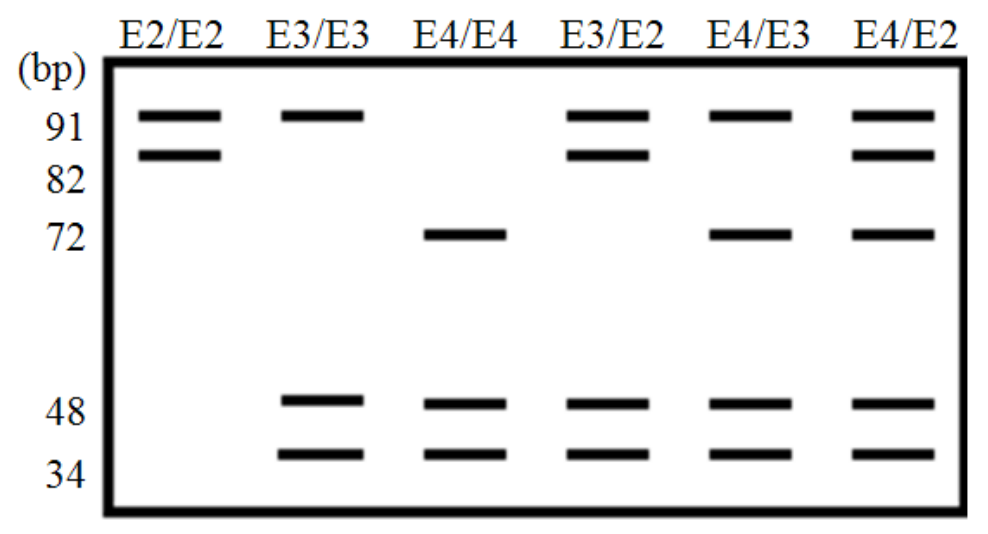

Fig. 2. ApoE restriction fragments for particular genotypes 
Jana Lisyova et al. / OnLine Journal of Biological Sciences 14 (1): 36-43, 2014

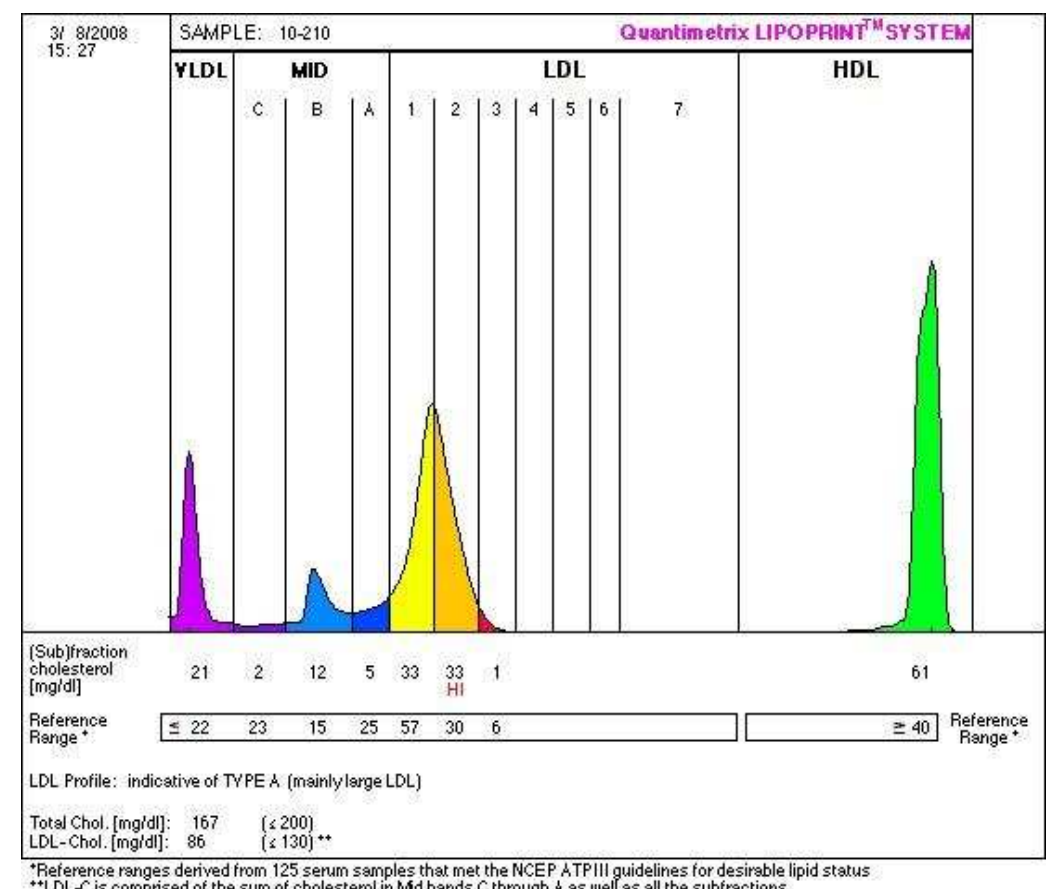

Fig. 3. Physiologic non-atherogenic lipoprint report (http://www.intechopen.com/source/html/39531/media/image2.jpeg)

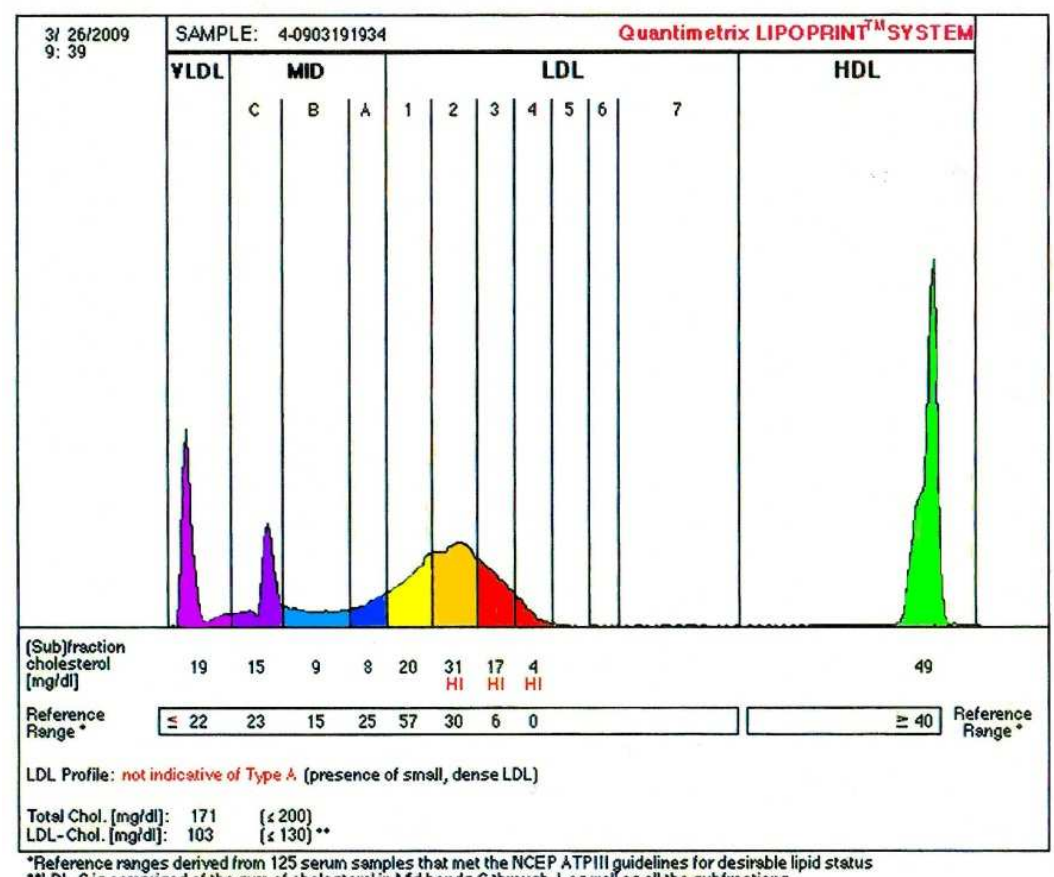

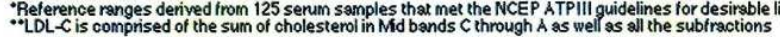

Fig. 4. Non-physiologic lipoprint report with risk of premature atherosclerosis (http://www.intechopen.com/source/html/39531 /media/image6.jpeg) 


\section{RESULTS}

During routine diagnostics, we identified a rare apoE mutation in a 51-year old male with severe short-term memory problems since 48 years of age. The moleculargenetic examination was recommended because of developing demetia with not known etiology (suspected AD). The proband's mother died of neurological disorder (suspected Creutzfeldt-Jakob or AD) in 62 years of age. Information about other first-relatives were not available.

The lipid parameters assayed by routine biochemical methods showed the following values: Total cholesterol $\left(4.67 \mathrm{mmol} \mathrm{L}^{-1}\right)$ and LDL cholesterol $\left(2.64 \mathrm{mmol} \mathrm{L}^{-1}\right)$ in reference ranges $\left(3.5-5.0 \mathrm{mmol} \mathrm{L}^{-1}\right.$ and $<4 \mathrm{mmol} \mathrm{L}^{-1}$, respectively), but a high levels of triacylglycerols $\left(2.98 \mathrm{mmol} \mathrm{L}^{-1}\right.$; ref. range $0.45-1.70$ mmol L $\left.{ }^{-1}\right)$ and low levels of HDL cholesterol (0.68 mmol L ${ }^{-1}$; ref. range 1.0-2.7 $\mathrm{mmol} \mathrm{L}^{-1}$ ).

A lipoprint report generated by Lipoprint System detected and quantified lipoprotein fractions and subfractions as stated below. Out of reference ranges were VLDL $\left(2.61 \mathrm{mmol} \mathrm{L}^{-1}\right.$; ref. range $\left.<1.22 \mathrm{mmol} \mathrm{L}^{-1}\right)$,
IDL1 $\left(1.50 \mathrm{mmol} \mathrm{L}^{-1}\right.$; ref. range $\left.<1.27 \mathrm{mmol} \mathrm{L}^{-1}\right)$, IDL2 $\left(0.94 \mathrm{mmol} \mathrm{L}^{-1}\right.$; ref. range $\left.<0.83 \mathrm{mmol} \mathrm{L}^{-1}\right)$, LDL3 $(0.38$ mmol L ${ }^{-1}$; ref. range $\left.<0.33 \mathrm{mmol} \mathrm{L}^{-1}\right)$ and HDL $(1.44$ mmol L ${ }^{-1}$; ref. range $>2.22 \mathrm{mmol} \mathrm{L}^{-1}$ ).

By molecular-genetic analysis (PCR-RFLP), we detected the presence of a rare $109 \mathrm{bp}$ fragment which does not appear in the common genotypes (Fig. 5). This finding indicates a loss of the restriction site (Hubacek et al., 2009).

A sequencing analysis revealed that both alleles carried typical apoE3 amino acid residues 112Cys (TGC) and 158Arg (CGC), but also a single-nucleotide substitution at amino acid position 136 (Arg $\rightarrow$ Cys) on one allele. This mutation lies at complementary DNA (cDNA) position 3817 and is located on the border within the putative LDL-receptor binding domain (Hubacek et al., 2009). Thus, we detected a rare mutation C3817T (p.Arg136Cys) on one allele and the common apoE3 isoform on the second allele (Fig. 6). This isoform is mentioned in literature as the apoE2* (Hubacek et al., 2009) and is characterized by the loss of restriction site at position 136.

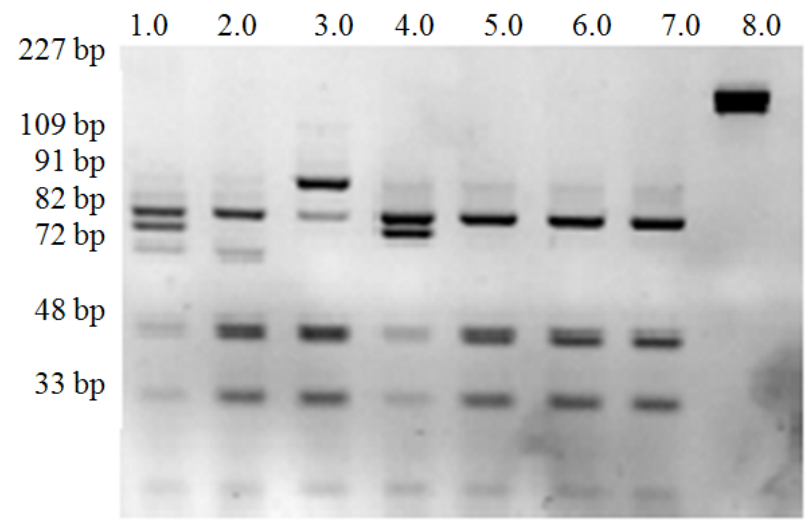

Fig. 5. PCR-RFLP of particular apoE genotypes. Lane 1- E4/2, lane 2- E3/4, lane 3- E3/2*, lane 4- E3/2, lane 5-7- E3/3, lane 8undigested DNA amplificate

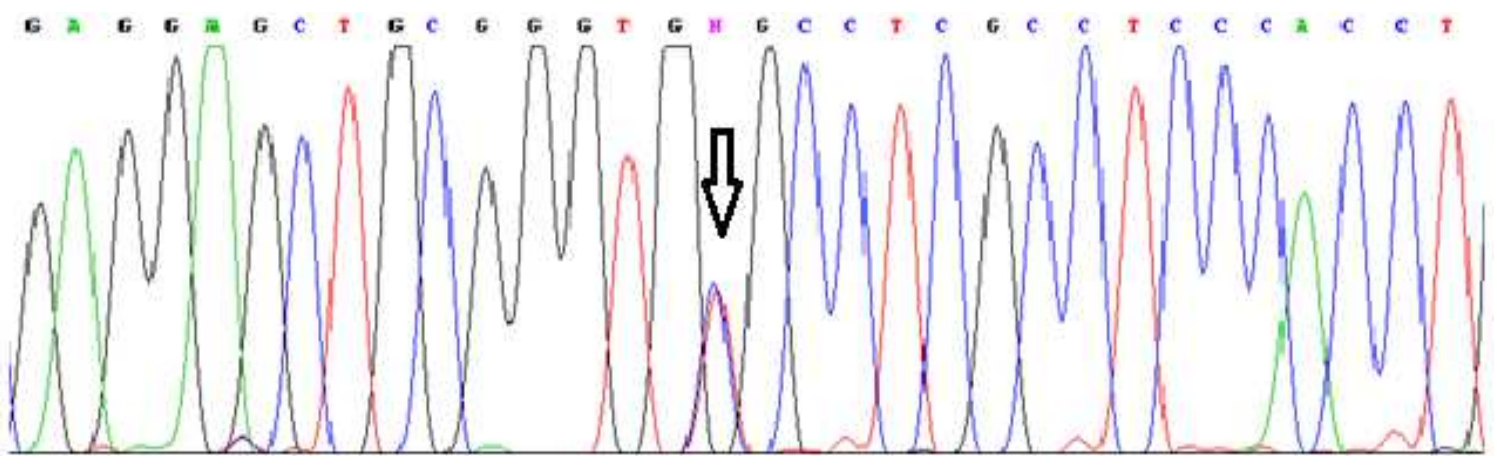

Fig. 6. Heterozygous apo E3/E2* profile 


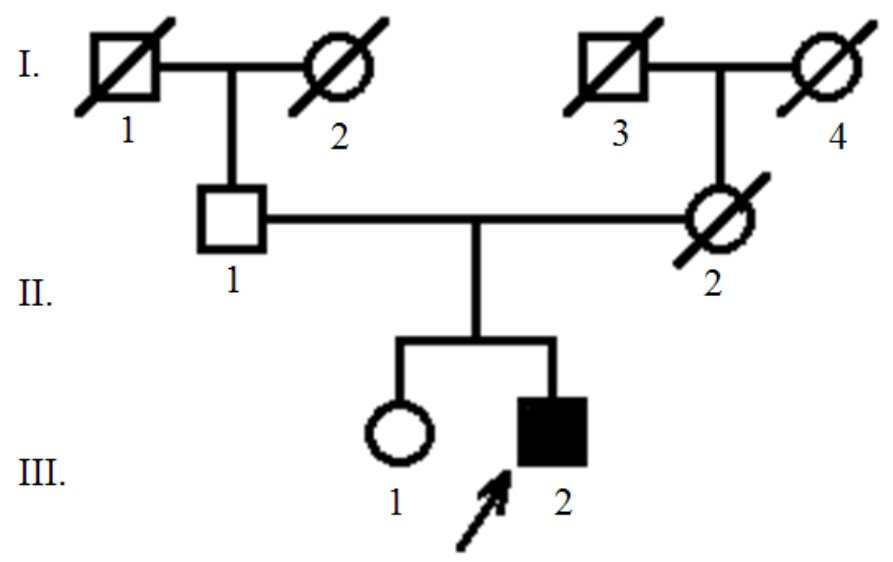

Fig. 7. Available pedigree of investigated proband

On our request, we admitted a proband's pedigree (Fig. 7) from Department of Medical Genetics (F. D. Roosevelt University Hospital, Banska Bystrica) with the partial medical history, only. We obtained the blood sample of his 82-year old father, too. According to all available data, the parents of proband's father died of tuberculosis (I.1) and myocardial infarction (I.2). The parents of proband's mother died of myocardial infarction (I.3) in 63 years of age and embolism (I.4) in 72 years of age. The proband's sister overcame a carcinoma of lung and uterus (III.1). By apoE genotyping of DNA sample of proband's father (II.1), we detected the same rare p.Arg136Cys mutation in heterozygous state (apoE3/E2*) but we received no information relevant to his health status, particularly to neurological diseases.

\section{DISCUSSION}

In proband, we have identified and confirmed by molecular-genetic methods a rare mutation of APOE gene. An estimation of detected genetic change and impact for given clinical finding is problematic. This time, neither molecular-genetic examination of family members nor data of parents's lipid profile is available. These specifications could be help us to interpret and estimate a prognosis of patient.

Through the population screening, Hubacek et al. (2008) detected twelve apoE p.Arg136Cys carriers. All of them had high Body Mass Index (BMI), elevated plasma lipids (with one exception) and six of them were on hypolipidemic treatment at the time of examination.
None of them suffered from CAD or had familial history of CAD. Only one carrier had the lipid profile typical for hyperlipidemia type III (HLP III). Thus, the hyperlipidemic pattern of these patients could be a secondary manifestation of high BMI and is not necessarily related to the presence of detected singlenucleotide substitution.

This mutation was also detected in two young survivors of myocardial infarction without remarkable dyslipidemia in both cases (Hubacek et al., 2009). Both were obese and smokers and one of them had strong positive family history of cardiovascular disease. The authors do not exclude that this mutation renders its carriers more sensitive to atherosclerosis in combination with other risk factors (e.g., obesity and smoking).

Our case report may open a discussion on possible linking of this rare genotype to neurological pathology and development of dementia. In backround of known apoE role in neurobiology, this consideration seems to be justified.

\section{CONCLUSION}

A missense APOE gene mutation p.Arg136Cys found in our patient seems to be related to neurological findings-short-term memory and dementia. On the other hand, it is problematic to associate this rare mutation with the typical clinical findings. APOE2* gene variant is very rare and consequently it is difficult to expect frequent studies evaluating statistical significance of this molecular change, in relation to phenotypic pattern. Descriptive approach and publication of case reports that 
evaluate the biochemical changes concerning lipoprotein profile and consequence on health status, related to the presence of apos 2* allele, are more expected. In the future, it would be necessary to perform targeted sequence analyses of APOE gene in patients with significant changes in lipid and lipoprotein profile, in association with the above-mentioned familial neurological problems with not known etiology. If we deliberate the knowledge about the impact of apoE genotypes on manifestation of $\mathrm{AD}$, we can predict logically that apoE2* isoform can represent a risk factor for this serious disorder.

\section{REFERENCES}

Bertram, L., Ch.M. Lill and R.E. Tanzi, 2010. The Genetics of alzheimer disease: Back to the future. Neuron, $\quad 68$ : 270-281. DOI: 10.1016/j.neuron.2010.10.013

Burman, D., A. Mente, R.A. Hegele, S. Islam and S. Yusuf et al., 2009. Relationship of the ApoE polymorphism to plasma lipid traits among South Asians, Chinese and Europeans living in Canada. Atherosclerosis, 203: 192-200. DOI: 10.1016/j.atherosclerosis.2008.06.007

Cedazo-Minguez, A., 2007. Apolipoprotein E and Alzheimer's disease: Molecular mechanisms and therapeutic opportunities. J. Cell. Mol. Med., 11: 1227-1238. DOI: $\quad 10.1111 / \mathrm{j} .1582-$ 4934.2007.00130.x

Coon, K.D., A.J. Myers, D.W. Craig, J.A. Webster and J.V. Pearson et al., 2007. A high-density wholegenome association study reveals that APOE is the major susceptibility gene for sporadic late-onset alzheimer's disease. J. Clin. Psychiatry, 68: 613618. PMID: 17474819

Crean, S., A. Ward, C.J. Mercaldi, J.M. Collins and M.N. Cook et al., 2011. Apolipoprotein E $\varepsilon 4$ prevalence in alzheimer's disease patients varies across global populations: A systematic literature review and meta-analysis. Dement Geriatr Cogn Disord, 31: 2030. DOI: 10.1159/000321984

Drenos, F. and T.B.L. Kirkwood, 2010. Selection on alleles affecting human longevity and late-life disease: The example of apolipoprotein E. PLoS ONE, 5: e10022. DOI: 10.1371/journal.pone.0010022
Elliott, D.A., G.M. Halliday and B. Garner, 2010. Apolipoprotein-E forms dimers in human frontal cortex and hippocampus. BMC Neurosci., 11: 2323. DOI: $10.1186 / 1471-2202-11-23$

Elmadbouh, I., Y. Elghobashy, E. Abd-Allah, A.-A. Reda and A. Fathe et al., 2013. Relationship of apolipoprotein E polymorphism with lipid profiles in atherosclerotic coronary artery disease. Egypt. Heart J., 65: 71-78. DOI: 10.1016/j.ehj.2012.11.002

Gafencu, A.V., M.R. Robciuc, E. Fuior, V.I. Zannis and D. Kardassis et al., 2007. Inflammatory signaling pathways regulating ApoE gene expression in macrophages. J. Biol. Chem., 282: 21776-21785. DOI: $10.1074 /$ jbc.M611422200

Gregorio, M.L., M.A.S. Pinhel, C.L. Sado, G.S. Longo and F.N. Oliveira et al., 2013. Impact of genetic variants of apolipoprotein $\mathrm{E}$ on lipid profile in patients with Parkinson's disease. BioMed. Res. Int., 2013: 641515-641515. DOI: 10.1155/2013/641515, PMID: 24175296

Hoefner, D.M., S.D. Hodel, J.F. O’Brien, E.L. Branum and D. Sun et al., 2001. Development of a rapid, quantitative method for LDL subfractionation with use of the quantimetrix lipoprint LDL system. Clin. Chem., 47: 266-274. PMID: 11159775

Hubacek, J.A., A. Peasey, H. Pikhart, P. Stavek, R. Kubinova et al., 2010. APOE polymorphism and its effect on plasma C-reactive protein levels in a large general population sample. Human Immunol., 71: 304-308. DOI: 10.1016/j.humimm.2010.01.008

Hubacek, J.A., R. Poledne, J. Pitha, M. Aschermann and H. Skalicka et al., 2009. Apolipoprotein E Arg136 $\rightarrow$ Cys in individuals with premature myocardial infarction. Folia Biol. (Praha), 55: 116118. PMID: 19545491

Hubacek, J.A., V. Adamkova, P. Stavek, R. Kubinova and A. Peasey et al., 2008. Apolipoprotein E Arg136 $\rightarrow$ Cys mutation and hyperlipidemia in a large central European population sample. Clin. Chim. Acta, 388: 217-218. PMID: 18326082

Irshad, M., R. Dubey, 2005. Apolipoproteins and their role in different clinical conditions: An overview. Ind. J. Biochem. Biophys., 42: 73-80. PMID: 23923565

Ping, G., B. Zhu, M. Jabasini, F. Xu and H. Oka et al., 2005. Analysis of lipoproteins by microchip electrophoresis with high speed and high reproducibility. Analytical Chem., 77: 7282-7287. PMID: 16285676 
Sando, S.B., S. Melquist, A. Cannon, M.L. Hutton and O. Sletvold et al., 2008. APOE $\varepsilon 4$ lowers age at onset and is a high risk factor for Alzheimer's disease; A case control study from central Norway. BMC Neurol., 8: 9-9. DOI: 10.1186/1471-2377-8-9

Sawle, A., M.K. Higgins, M.P. Olivant and J.A. Higgins, 2002. A rapid single-step centrifugation method for determination of HDL, LDL and VLDL cholesterol and $\mathrm{TG}$ and identification of predominant LDL subclass. J. Lipid Res., 43: 335-343. PMID: 11861676
Ward, H., P.N. Mitrou, R. Bowman, R. Luben and N.J. Wareham et al., 2009. APOE genotype, lipids and coronary heart disease risk: A prospective population study. Arch Intern. Med., 169: 14241429. DOI: 10.1001/archinternmed.2009.234

Zhong, N., K.H. Weisgraber, 2009. Understanding the association of apolipoprotein E4 with alzheimer disease: Clues from its structure. J. Biol. Chem., 284: 6027-6031. DOI: 10.1074/jbc.R800009200 